Journal of Computer Science 8 (9): 1531-1540, 2012

ISSN 1549-3636

(C) 2012 Science Publications

\title{
A Computational Trust Model for Collaborative Ventures
}

\author{
${ }^{1}$ Mohd Mokhtar and ${ }^{2}$ Weigang Wang \\ ${ }^{1}$ Center of Computer Science, Faculty of Information Science and Technology, \\ Universiti Kebangsaan Malaysia, 43600 Bangi, Selangor, Malaysia \\ ${ }^{2}$ Manchester Business School, The University of Manchester, United Kingdom
}

\begin{abstract}
Problem statement: The conceptual notion of trust and its underlying computational methods has been an important issue for researchers in electronic communities. While the independent trust evaluation is suitable in certain circumstances, such unilateral process falls short in supporting mutual evaluation between partners. Perceived reputation, the depth and breadth of trust, Trust Perception (TP), Repeat Collaborators at a Threshold (RCT) and a collective trust index (c index) have all been defined to specify the optimal trust criteria. Approach: By taking the evaluator's own trust level as a threshold to identify compatible partners, a mutual balance between excess and deficiency in trust has been addressed. Since the number of repeated collaborations which signify retested confidence is more straightforward to capture than the manually provided feedback ratings, we have developed computational definitions for the above-mentioned concepts. Results and Conclusion: The results from the experiments based on the eBay dataset shows that the $\mathrm{c}$ index can be used to classify PowerSellers into normally distributed and comprehensible categories that can facilitate mutual evaluation.
\end{abstract}

Key words: Trust, Reputation, $\mathrm{c}$ index, $\mathrm{h}$ index

\section{INTRODUCTION}

Since the beginning of time, as human beings we have always been trying our very best to achieve the highest possible feat in whatever we do. Regardless of the cost or consequence, we crave for praise in building the tallest, the highest or the longest of almost everything that we can think of. We constantly have this impression that the further and higher we go, the better we will be. Therefore, it is not surprising that this approach has also affected the way we make our everyday decisions, including in trust and partner selections. This work tries to provide a fresh view of dealing with trust and trust-based partner selection where we believe that, under specific circumstances, there will be situations in which the most highly regarded partner may not be the best choice.

Before we go further into the discussions, we first need to clarify the notion of trust in this particular work due to the connotations that it may have in various situations. While there have been almost no disputes from the scholars on the importance of trust across various fields (Sabater and Sierra, 2005), it is however, difficult for them to agree on a universally recognized definition (Marsh, 1994; McKnight and Chervany, 2001).
Conceptual confusion on trust makes comparing one trust study to another a complicated process and rather difficult to define (McKnight and Chervany, 2001). In general, there are two main methods for managing trust decisions, namely policy and reputation. These two different techniques correspondingly describe the "strong" approach that is based on sets of digital credentials and the "soft" sociological approach that is based on reputation information gathered in community (Bonatti et al., 2005; Ramchurn et al., 2004a; 2004b) which usually equated to a numerical measurement. In this study, we decided to focus on the nature of trust that is based on the latter approach and we have adapted the concept of trust (Hosmer, 1995) which we believe captures many features of the definition of trust that we are focusing on:

"Trust is the reliance by one person, group, or firm upon a voluntarily accepted duty on the part of another one person, group, or firm to recognize and protect the rights and interests of all others engaged in a joint endeavour or economic exchange".

We will begin by explaining the concept of optimal trust through some examples, definitions and its relation to the collaborative trust framework. Then in we continue to discuss and describe all the elements involved in an optimal trust model; this is done along

Corresponding Author: Mohd Mokhtar, Center of Computer Science, Faculty of Information Science and Technology, Universiti Kebangsaan Malaysia, 43600 Bangi, Selangor, Malaysia Tel: +60389216665 


\section{J. Computer Sci., 8 (9): 1531-1540, 2012}

with its definition, examples, formulas and selection method. Finally, we discusses the eBay community as a case study, before we conclude this work in the final section.

The concept: In our effort to look for the different ways to use trust and reputation in assessing the trustworthiness of a partner, we have taken the liberty of exploring a novel concept of optimal trust (Wicks et al., 1999). In order to explain the structure of optimal trust, this particular work has highlighted the fact that we should not just focus on trust as it is, but that we should also theorize on the notion of optimal trust. In that study, they used Aristotle's ethics that focus on finding what he described as the "golden mean" between excess (overinvestment in trust) and deficiency (underinvestment in trust), as a conceptual theory to support the notion of optimal trust (Wicks et al., 1999).

In order to provide a stronger conceptual basis for further discussion on this notion, we need to grasp the concrete meaning of "optimal". According to the Oxford dictionary (OD, 2009), the word optimal is described as something: "best or most favourable". At the same time, the term can also be described as: the "most desirable possibility under a restriction expressed or implied" (Dictionary.com. 2009). However, we are more inclined to consider optimal as an action to obtain the desirable result using the right level of efforts.

Therefore, when we bring this optimal concept into a trust perspective, we can then define optimal trust as: A trusting action that is mutually acceptable by corresponding parties that is autonomous from the perception of the community. In other words, collaborators can mutually trust each other even if they are not the most popular in the community.

For that reason, looking for a collaboration partnership is a mutual selection process. In the real world, a realistic and mutually acceptable match is often found at a level that both parties belong to. Sadly, the Cinderella-kind story is only a fairy tale. For most people, it is an unrealistic dream. As an example, when an unknown business company looks for a partner; it may wish to find the best or a well-known partner. However, in the end, it is more likely that it would end up with one with a relatively similar standing as itself in that business. Based on common sense knowledge like this, we have derived a hypothesis.

Hypothesis: Most collaborators would prefer to select highly reputable partners; however, the majority of their close collaborators are those near to their own reputation standing.
There are two important criteria in this hypothesis; first is the preference of collaborators to seek a popular partner. This is actually not uncommon in the social concept of reputation; however, the second part of this hypothesis is not as it typically transpires that a selection process will require further exploration and verification. Accordingly, we found that most of the earlier works in this area accept that these "track records" or reputation are a very useful way to assess the trustworthiness of a potential partner. The only difference would be the way this record is gathered, processed and interpreted. Furthermore, there is a balance between the cost and benefits of having a good reputation (Kreps and Wilson, 1982) and revealing this mutual level of trust will be the challenge in our attempt to apply this concept in a trust model.

Unfortunately, most of the time we do not see trust and reputation as something tangible and therefore we rarely notice the cost of building such a level of trust. Hence, we cannot really see the bottom or the limit of trust or even the need to have one. As a result, the cost of building or acquiring such trust is often overlooked and underestimated. That is why, to help us expand and deliberate on this concept that we introduced, we have constructed a collaborative trust framework (Mokhtar et al., 2007), where we define collaboration as (Winer and Ray, 1994):

"Collaboration is a mutually beneficial and welldefined relationship entered into by two or more individuals to achieve results that they are more likely to achieve together rather than alone".

Based on this framework, we reasoned that unilateral dependency on its own is insufficient to determine the mutual partner; hence, we need to consider bidirectional dependencies (or interdependencies) between partners (Mokhtar et al., 2007). Our view on this is shared in the concept of optimal trust (Wicks et al., 1999) that we explained earlier, where they consider the dependency between partners as an essential criterion for creating the theory on trust. Besides this, similar work on bilateral negotiation by Brainov and Sandholm (1999), also suggested that if the level of trust is mutual, then the social welfare, the amount of trade and the utility functions can also be maximized.

As the interdependency between partners will not be able to develop within a single instance, but is instead developed through a series of repeated collaboration between the partners ( $\mathrm{Yu}$ and Singh, 2002), we need a trust model that is capable of capturing such actions. Moreover, through these repeated (or re-tested) interdependencies, the confidence and mutual trust between partners will further develop. 


\section{J. Computer Sci., 8 (9): 1531-1540, 2012}

By distinguishing the importance of the interdependencies in trust and collaboration, the framework helps us to deliberate this connection and the potential use of this trust concept without losing the applicability of this model in any other area. Thus through this framework, we have managed to identify the key principle that we can apply to the trust model.

The trust model: We have so far covered the concept of optimal trust and the distinctive approach that it can bring to the community. We now continue to explain the need to have such concept and the key elements of the collaborative trust framework and how we can utilize the above-mentioned concept to evaluate potential collaborative partners. In order to develop the optimal trust concept into a computational trust model, we need to design the main components of the optimal trust model and then explain how these different components facilitate the whole process of identifying potential partners through different types of trust measurements.

Trust components: First, we present a set of $n$ collaborators in a virtual community as $A=\left\{a_{1}, a_{2} \ldots\right.$ $\left.a_{n}\right\}$ that signifies a common form of virtual ventures between different entities. Although by default the collaborators or partners in our model refer to members in a human community, the aim is for this model to manage collaborative reputation in large-scale distributed systems such as multi agent, Peer-To-Peer (P2P), grid or mobile. In order to establish such collaboration, first the initiator $\mathrm{a}_{\mathrm{ev}}$ needs to evaluate each potential collaborator $\mathrm{a}_{\mathrm{pc}}$ in the community. At the same time, to decide whether to accept an invitation to collaborate or not, the potential collaborator will also evaluate the collaboration initiator, where the role will be reversed.

Direct Trust (DT): The Direct Trust of a person towards a partner is reflected by the accumulated personal experience the person has towards that partner. Such personal feeling has often been measured using specific forms of feedback based on the performance of the partner. However, existing feedback systems, such as those that require users to provide positive and negative feedback scores explicitly, do not provide sustained incentives for the users to provide such feedback on every instance of their collaboration and to behave honestly over time (Fan et al., 2005). This leads us to consider that a repeated collaboration between two collaborators is a better mechanism to gather such information that operates as an implicit positive feedback to the previous collaboration.

This value indicates what that partner personally "thinks" about the other partner by using the collaboration rate of recurrence as a measuring scale. It is one of the distinct features in this trust model when compared to any other trust models that have been previously proposed; where we intuitively presume that a collaborative partner will continue to collaborate with a specific partner so long as no major negative experience that cannot be reconciled occurs between them. If any serious dispute starts to happen between any partners, then future collaborations will likely be discontinued (or put on hold) until both parties can reconcile the current problem.

At the point of a successful reconciliation, both parties will agree to "forget" the dispute that they just had in their last collaboration by agreeing to the reconciliation package and continuing with the possibility of having future collaboration. So the more collaboration that can be performed without having to reconcile with a specific partner, the more trustworthy the partner is perceived by the evaluator.

In order to measure this, we take the number of collaborations between two collaborators as their implicit positive feedback scores. Such an implicit positive feedback score out of a specific collaboration may be cancelled or offset in a separate record-keeping system named the reconciliation process, by an explicit feedback provided by the partner of that collaboration.

Hence, the DT value of an evaluating partner $\mathrm{a}_{\mathrm{ev}}$ on a potential collaborator $\mathrm{a}_{\mathrm{pc}}$ can be defined as:

$$
\operatorname{DT}\left(\mathrm{aev}_{\mathrm{ev}}, \mathrm{a}_{\mathrm{pc}}\right)=\frac{\mathrm{n}\left(\mathrm{aev}_{\mathrm{ev}}, \mathrm{a}_{\mathrm{pc}}\right)-\mathrm{r}\left(\mathrm{aev}_{\mathrm{ev}}, \mathrm{a}_{\mathrm{pc}}\right)}{\mathrm{n}\left(\mathrm{aev}_{\mathrm{ev}}, \mathrm{a}_{\mathrm{pc}}\right)}
$$

where, $n\left(a_{e v}, a_{p c}\right)$ is the total number of collaborations between $\mathrm{a}_{\mathrm{ev}}$ and $\mathrm{a}_{\mathrm{pc}}$ and at the same time, $\mathrm{n}\left(\mathrm{a}_{\mathrm{ev}}, \mathrm{a}_{\mathrm{pc}}\right)$ is also the number of implicit positive feedback between them; while $r\left(a_{e v}, a_{p c}\right)$ refers to the number of explicit reconciliation $\mathrm{a}_{\mathrm{ev}}$ produced toward $\mathrm{a}_{\mathrm{pc}}$. A reconciliation may be cancelled (i.e., reset to 0 ) by the evaluator before their next collaboration if the issue which caused such negative view is reconciled with satisfactory corrective actions.

Reconciliation: Whenever any dispute occurs between collaborative partners, then either party will have the option of filing a reconciliation report. Studies show that a reputation system does not alleviate unintentional online offences; however, if the offender is given the opportunity to repair his action then the victim's trust can be restored (Vasalou et al., 2008). Therefore, the core element of this reconciliation process will consist of the main disputes between the collaborators, along with the recompense package requested for that particular report. For example, if a collaborative partner is not satisfied 


\section{J. Computer Sci., 8 (9): 1531-1540, 2012}

with the quality of the product supplied by the other partner, then a reconciliation report is made on the partner stating in detail the nature of complaints together with the recompense package such as providing a replacement or modifying an existing product.

Once that corresponding partner receives this report, an investigation will be made and the partner can either choose to accept the requested reconciliation package or renegotiate the package. At the same time, new collaboration can be performed in parallel with any reconciliation complaint. Reconciliation that occurs between two collaborating partners is denoted by $r$ $\left(\mathrm{a}_{1}, \mathrm{a}_{2}, \mathrm{c}, \mathrm{p}, \mathrm{s}\right)$ where $\mathrm{r}$ indicates the unique reconciliation identification number, $\mathrm{a}_{1}, \mathrm{a}_{2}$ represent the two parties involved in the dispute, $c$ registers out the nature of the complaint and $p$ lists down the recompense package. Meanwhile $s$ simply represents the status of the complaint, either unsolved or solved. Ideally, there will be a central body that can keep track of all the reports produced in a community.

Here we shall limit our discussion by assuming that the necessary mechanism has already been retained to evaluate a particular partner's performance based on the outcome of the previous collaboration. Further examples of how this could be done can be found in previous works (Ramchurn et al., 2004a; 2004b; Teacy et al., 2006) that have given additional focus on this issue.

By doing things this way, we have not only managed to solve disputes among the collaborative partners that negative feedback could not, but we have also managed to determine how well a partner reacted to a conflict. Furthermore, a reconciliation method will be able to gather a larger amount of data with significantly less overhead cost as only around 50-60\% of the buyers and sellers actually leave any feedback and $99.1 \%$ of these feedbacks are all positive (Resnick and Zeckhauser, 2002).

Alternatively, we could also choose not to collect any of the information from the reconciliation processes altogether, in which we have to assume that future collaboration will continue between partners so long as the experience stays positive and all the reconciliations between them are achieved. Hence, a successful reconciliation is regarded a positive remark that strengthens the mutual understanding between partners until any irreconcilable issue actually stops the partnership indefinitely. In doing so, we would be able to rely completely on automatically logged data without the need to have a single manually entered feedback. Subsequently, we will use another personal experience based trust measurement, Trust Depth (TD) to replace DT. We will demonstrate how this can be done in the close collaborator case study.
Perceived Reputation (PR): The REGRET reputation model (Sabater and Sierra, 2001) broadly defines reputation as: "opinion or view of one about something". Based on this, we introduce the concept of Perceived Reputation (PR) as the average means of the DTs gathered from every collaborator in the community that in a way reflects the general trust perception of a particular partner; therefore, the PR $\left(\mathrm{a}_{\mathrm{pc}}\right)$ of a potential collaborator is defined as:

$$
\operatorname{PR}\left(\mathrm{a}_{\mathrm{pc}}\right)=\sum_{i=1}^{\mathrm{m}} \frac{\mathrm{DT}\left(\mathrm{a}_{i}, \mathrm{a}_{\mathrm{pc}}\right)}{\mathrm{m}}
$$

where, $\mathrm{i}=1,2, \ldots, \mathrm{m}$ and $\mathrm{m}$ is the total number of collaborators in a community that have had direct collaborative interaction with $\mathrm{a}_{\mathrm{pc}}$. If $\mathrm{m}=0$, i.e., no direct collaborative interaction with $a_{p c}$ can be found, then the PR value will be 0 .

This PR value will be updated whenever a collaborator performs any form of collaborative action with others. Therefore, from the point of view of the entire community the better a collaborator collaborates with the others in the community, the better the general PR that collaborator will have. That gives us an approximation of the general trust perception of a particular collaborator; although this simple and generalized view is often adequate, it can at times fall short in reflecting the actual collaboration recurrence between partners. In order to tackle this problem, we introduce the concept of Trust Depth and Trust Breadth.

Trust Depth (TD) and Trust Breadth (TB): The definition for Perceived Reputation (PR) is an average ratio of all DT values provided by all collaborators towards a specific partner. It is simple to understand and use. However, the DT and PR do not typically reflect how deep a trust is (in terms of recurrence) and how widely a trust is distributed across a community (in terms of the number of different partners). For instance, the highest PR value 1 may come out of one collaborative venture or 1000 collaborative transactions (i.e., 1/1 or 1000/1000). In the case of the 1000 collaborations, it may happen with one collaborator or with many collaborators. Thus, to have a more finegrained and meaningful differentiation, we define the concepts of trust depth and trust breadth as follows.

We define Trust Depth (TD) that the collaborator received from a potential collaborator, as the number of Pure Repeated Collaboration (PRC) he or she performed with that particular collaborator. Pure repeated collaboration PRC $\left(\mathrm{a}_{\mathrm{ev}}, \mathrm{a}_{\mathrm{pc}}\right)$ is defined as the total number of collaborations minus the total number of reconciliation $n\left(a_{e v}, a_{p c}\right)-r\left(a_{e v}, a_{p c}\right)$; If this value is 


\section{J. Computer Sci., 8 (9): 1531-1540, 2012}

less than 0 , we define the PRC $\left(a_{\mathrm{ev}}, \mathrm{a}_{\mathrm{pc}}\right)=0$. This PRC value is what we call the efficient collaborations, which are performed without having any disputes between the partners. Therefore:

$$
\begin{aligned}
& \operatorname{PRC}\left(\mathrm{aev}_{\mathrm{ev}}, \mathrm{a}_{\mathrm{pc}}\right)=\mathrm{n}\left(\mathrm{a}_{\mathrm{ev}}, \mathrm{a}_{\mathrm{pc}}\right)-\mathrm{r}\left(\mathrm{a}_{\mathrm{ev}}, \mathrm{a}_{\mathrm{pc}}\right) \text { if } \geq 0 \\
& \text { or } \\
& \operatorname{PRC}\left(\mathrm{aev}_{\mathrm{ev}}, \mathrm{a}_{\mathrm{pc}}\right)=0 \quad \text { if }<0
\end{aligned}
$$

This PRC value can also be used as a simple trust measurement that is able to evaluate repeated collaboration between collaborators. On the other hand, we define Trust Breadth (TB) that a collaborator has in a community as the total number of positive partners (i.e.: those with a PRC equal to or greater than 1).

In the above definitions, TD indicates the number of repeat or retested trustworthy collaboration with a collaborator, while TB indicates how widely such trustworthy collaboration has occurred with different collaborators. The TB concept is somewhat similar to the trust score concept used by eBay, 2009. However, the eBay trust score and the eBay positive feedback concepts do not fully take into account repeat transactions from the same partner as reflected by our $\mathrm{TD}$ and $\mathrm{TB}$ concepts.

Collective and individual trust: With the inclusion of different trust components in DT, PR, TD and TB, we have provided additional information that can help us in assessing one's reputation and trustworthiness. However, more parameters make the comparison process on trust and reputation more complicated to compute and employ. We categorize the trust measurements into collective (c index and $\mathrm{RC}_{\mathrm{T}}$ ) and personal (TP) trust.

Collaboration index (c index): In order to have a single collective measurement that is robust and accurate, we decided to follow the idea of the $\mathrm{h}$ index (Hirsch, 2005) to define an index, known as the c index, to measure the collaborative trust that collaborators gained from a community. In order to help us explain what the $c$ index is, we will first introduce the $h$ index. In his study, Hirsch proposed a simple yet robust citation index that quantifies the research output of individual scientists. The $\mathrm{h}$ index is defined as: "A scientist has index $h$, if $h$ of his/her Np papers have at least $\mathrm{h}$ citations each and the other $(\mathrm{Np}-\mathrm{h})$ papers have fewer than h citations each" where $\mathrm{Np}$ is the number of papers published over $n$ years (Hirsch, 2005).

In a similar way, the $\mathrm{c}$ index for a collaborator- $a$ is defined as: $a$ has index c if $a$ has $c$ collaborators each of whom has given $a$ at least $c$ pure repeated collaborations and each of other $(\mathrm{Nc}-\mathrm{c})$ collaborators has provided less than $c$ pure repeated collaboration to $a$ where $\mathrm{Nc}$ is the total number of all collaborators of $a$.

As an example, if collaborator- $a$ received $8,7,4$ and 2 pure repeated collaborations from $b, c, d$ and $e$ respectively; the $\mathrm{c}$ index for collaborator- $a$ will be 3 . This is because 3 out of the 4 collaborators have given at least 3 PRC towards collaborator- $a$ and the rest of $(4-3=1)$ collaborator have provided less than 3 PRC.

Figure 1 shows the $c$ index from a plot of decreasing collaborations for numbered collaborators. Here the vertical axis of the number of collaborations reflects the TD where each positive unit represents repeated collaborations by an individual collaborator, while the horizontal axis of each unique positive collaborator reflects the collaborator's TB.

The trust measurement using the $\mathrm{c}$ index is much more robust than that of PR, as it is much more difficult to be manipulated by others when compared to PR. For instance, a high number of collaborations from one friendly collaborator may raise PR to some extent, but the $\mathrm{c}$ index will not be significantly affected. This is because a series of scheming manipulations (Dellarocas, 2000) will not be able to change the c index directly, as it will take a group of collaborators that is larger than the index itself to significantly change anything-thus making it much more robust though still maintaining a simple yet efficient index.

The computation method of $\mathrm{c}$ index is in a way similar to that of the $\mathrm{h}$ index as it uses the same conceptual elements. However, unlike the $\mathrm{h}$ index that always grows, the $\mathrm{c}$ index of a collaborator may drop as the collaborator's PRC may drop if negative feedback to the collaborator happens. At the same time, the c index also differs from the feedback score in the eBay system which only measures the breadth of trust; the $c$ index takes both the depth and breadth of trust into consideration. Meanwhile, eBay has recently changed their system to incorporate repeat feedback as part of eBay's wholesale changes in 2008. This move suggests the importance of including genuine repeat transactions in the system.

Then again, as the feedback scores in eBay are based on the manually entered feedback, transactions that left without a feedback are omitted. This is the major difference between such a data collection technique and the repeat satisfactory collaborations that are captured using the $\mathrm{c}$ index which can be achieved without the need for explicit feedback. Nevertheless, the negative feedback in the reconciliation process still has to be provided for explicitly. 
J. Computer Sci., 8 (9): 1531-1540, 2012

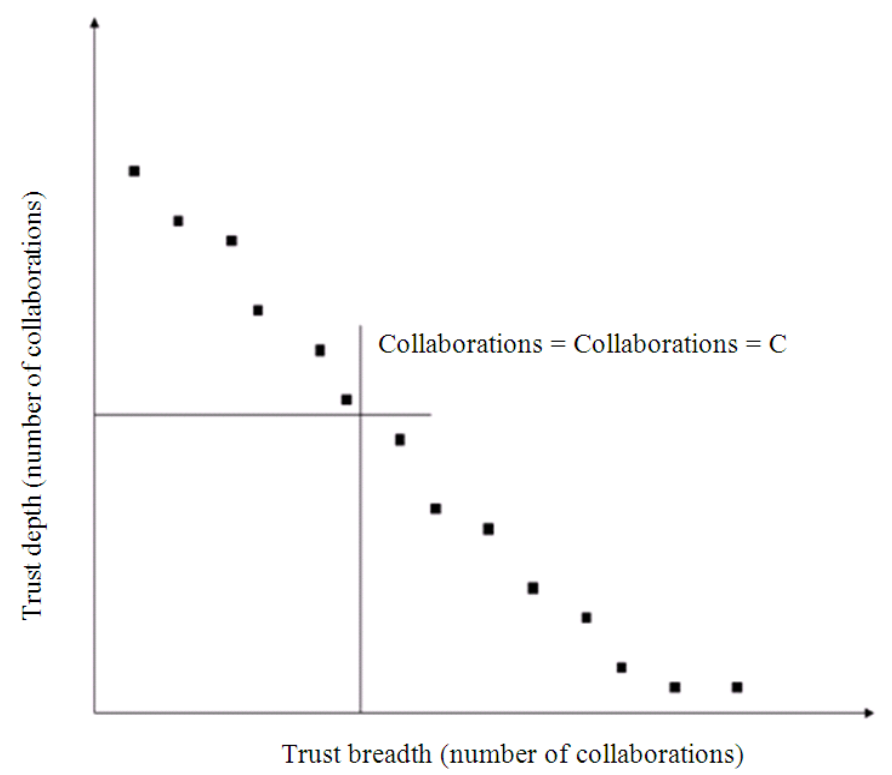

Fig. 1: C Index from a plot of decreasing collaborations for numbered collaborators

Repeat collaborators at a threshold: As we reflect on the usability of the $c$ index, it is clear that upon introducing a robust measurement, we have also made one's c index difficult to grow as it only grows when most existing collaborators continue their collaboration with that same partner. In a situation where there is a further need to compare such as in open and large environments where repeated interactions can be difficult to find, we require a different approach by maintaining a threshold that is above a specific repetitive performance level. In view of this, we have introduced the concept of Repeat Collaborators at a TD Threshold $\left(\mathrm{RC}_{\mathrm{T}}\right)$ to do just this.

This is where $\mathrm{RC}_{\mathrm{T}}$ is different from the $\mathrm{c}$ index because unlike the $\mathrm{c}$ index, $\mathrm{RC}_{\mathrm{T}}$ identifies a specific repetitive (TD) level and measures the number of collaborators at that level. Hence, this makes it easier to grow than the $\mathrm{c}$ index. For example, a collaborator who has 10 different partners with $\mathrm{c}$ index $=2$, would need a third collaborator to collaborate with for at least three times and for the earlier two collaborators that were included in the $\mathrm{c}$ index measurement to also have at least one more collaboration each. On the other hand, for $\mathrm{RC}_{\mathrm{T}}$ we will only be assessing the number of collaborators that is above a pre-defined threshold level. For instance, at a threshold level 2, a collaborator with c index of 2 may have an $\mathrm{RC}_{2}$ of 5 if the person has 5 collaborators who have provided a $\mathrm{TD}>=2$. By doing so, we are still evaluating the repetitive performance of a particular collaborator, but only at a specific TD level.

Trust Perception (TP): Trust Perception (TP) is defined as an individual's overall assessment of another collaborator that combines the evaluator's DT and the opinions that come from a community (PR). The weight of these two values corresponds to the requirement at any particular time, which can be determined accordingly. As the purpose of a trust evaluation is often to see if a potential partner would be suitable enough for performing a specific collaboration, the trust perception value of a collaborator towards another collaborator may differ when the collaboration to be performed differs.

By taking these into account, we define TP as:

$$
\mathrm{TP}(\mathrm{a} \text { ev, } \mathrm{a} \text { pc })=\alpha \mathrm{DT}(\mathrm{a} \text { ev }, \mathrm{apc})+\beta \operatorname{PR}(\mathrm{a} \mathrm{pc})
$$

where, DT $\left(a_{e v}, a_{p c}\right)$ is the evaluator's direct trust pertaining to the potential collaborator; $\operatorname{PR}\left(a_{p c}\right)$ is the perceived reputation of the potential collaborator in the community and $\alpha$ and $\beta$ are weighting criteria for these two components $(0 \leq \alpha \leq 1,0 \leq \beta \leq 1$ and $\alpha+\beta=1)$. Thus, $\alpha$ can be regarded as how well the two collaborators know each other (based on DT), while $\beta$ on the other hand reflects the community's perception.

Based on the study previously carried out by Ramchurn et al. (2004a; 2004b), the more interactions we make with a specific partner, the more reliance we will have on our own personal confidence (direct trust) because it is judged to be more accurate than information gathered from other sources (that might be subject to noise). Therefore, the better two collaborators know each other, the more weight they will place on their own experience (i.e., DT); in the reverse circumstance, more weight will be placed on others' option (i.e., PR). 


\section{J. Computer Sci., 8 (9): 1531-1540, 2012}

Unlike the $\mathrm{c}$ index and $\mathrm{RC}_{\mathrm{T}}$ that capture the collective trust experiences in the community, this combination of an individual and collective trust component is more flexible in situations when firsthand trust experience is important. This also signifies the social norm that our first-hand experiences sometimes have a big impact on our trust perception. It also reflects the fact that we often cooperate with some people whose reputation in a community is not as high, but our mutual trust is high based on our successful collaboration in the past on some common tasks. However, when the collaborators do not know each other (i.e., $\mathrm{n}\left(\mathrm{a}_{\mathrm{ev}}, \mathrm{a}_{\mathrm{pc}}\right)=0$ ), $\alpha$ can therefore can be set to 0 and $\beta$ to 1 .

Selection method: Before going through the selection approach that is going to be implemented in greater detail, we will discuss some of the key features underlying the selection method. The partner selection method is based on the level of mutual trust between potential collaborators. Based on the collaborative trust framework (Mokhtar et al., 2007), this mutual trust is reflected by the level of proximity towards a chosen trust measurement value (i.e., the $\mathrm{c}$ index) that a collaboration initiator shares with his or her potential partner.

For that reason, in this selection method, the closer these values are, the higher the chances that the collaboration invitation will be accepted by the partner because this either indicates that their trust standing in a community is at the same level, or they share a compatible mutual feeling of trust towards each other. In the latter case, it is necessary to exclude any case of mutual distrust demonstrated by the negative reconciliation feedback they had given to each other in their past collaborations. The value has to be either at a significant level or if low, is not due to such negative feedback, but because their collaboration history has just started.

Potential collaborating candidates can be identified by examining whether they have a common goal, complementary skills and an agreement on the level of shared risk that relates to the task to be performed. The computational method that we describe in the following assumes that the first stage has been completed, which can be done through some negotiation and data mining process and the second step of the selection phase starts by giving the collaboration initiator a list of potential collaborating candidates in a community. In short, we assumed that the potential partners are selected from those who already share a common goal, have the skills needed for the task at hand and share the same level of risk with the evaluator.

\section{MATERIALS AND METHODS}

The principal idea of the selection process is to identify partners who share a compatible and significant level of trust between them. The selection process starts with computing the collaboration initiator's own c index value and the $\mathrm{c}$ index values of all the potential partners. Then, taking the collaboration initiator's own c index value as a threshold to identify partners at the same trust level (i.e., if $\mid \mathrm{C}$ (a)-C(b) $\mid \leq$ delta, say delta $=1$, then collaborator- $a$ and collaborator- $b$ are considered at about the same level). Noticeably, as the number of collaborators and the number of collaborations increases, the $\mathrm{c}$ index will also increase. This allows the collaborator to collaborate in a different level.

If we take any sports for example, the majority of these sports will have their own league which differentiates between teams or players at different levels. Players may wish to play in a higher level; however he will often compete with those at similar levels. Hence, if collaborations are performed among lower level collaborators, that does not necessarily mean that the collaborators are not trustworthy (or cannot play the game). When they excel at one level, they are then moved into a higher level. In a similar way, this selection method supports and promotes such typical social concepts.

This flexibility in criteria configuration that uses the evaluator's own individual or collective trust measurement as a threshold to identify compatible partners, which reflects the optimal trust principle of identifying the "golden mean" between excess in trust and deficiency in trust based on the need at hand (Wicks et al., 1999) is the fundamental concept for this model.

eBay case study: We decided to choose eBay as the dataset for a preliminary case study mainly for two reasons: First, we would like to analyse the applicability of the trust model using available dataset that can be tested directly. Secondly, the main aim for this part of the study is to illustrate how different levels of repeated interactions can be used as a basis for the notion of classifying potential partners by using a feedback-type reporting system such as that being used in eBay.

Table 1: The eBay dataset feedback score

\begin{tabular}{lrc}
\hline Type & Score & Percentage \\
\hline Positive & 75569 & 99.1 \\
Negative & 237 & 0.3 \\
Neutral & 402 & 0.5 \\
Total & 76208 & 100.0 \\
\hline
\end{tabular}




\section{J. Computer Sci., 8 (9): 1531-1540, 2012}

Background: The case study data we collected was completely based on the feedbacks given towards eBay PowerSellers in the period of 12 months between June 2007 and July 2008. This is in fact the maximum period providing detailed feedback between buyers and sellers that we could actually trace online. We chose the toner cartridge auction by eBay PowerSellers because we wanted a sample of a dataset that came from actively trading sellers, where a repetitive string of good services would be deemed as something very important to both buyers and sellers that in some way signifies the mutual dependence between buyers and sellers.

We used a web scraper program named Mozenda (2008) to gather all the feedback dataset from the 31 PowerSeller shops. Each eBay member has its own feedback page where all the feedback information is presented. Every time a transaction takes place between eBay members, the buyer and seller will rate each other by giving positive, negative or neutral feedback that will in turn affect the overall feedback score and the percentage of positive feedback. The web scraper will then go through each page and collect all the feedback information.

The dataset: In each dataset, we focus on collecting three main details; (i) from where the feedback was received (ii) the date, when the feedback was given and (iii) time. All the information was compiled straightaway into database tables. All together, we managed to collect 76,208 unique feedbacks, which were then classified into individual tables in a single database. The preliminary data is presented in Table 1.

\section{RESULTS AND DISCUSSION}

The repetitive aspects from the ongoing buyerseller relationship were then assessed by examining the feedback histories on the set of 31 PowerSellers in the dataset. Overall, these sellers accumulated 76,208 feedbacks between them in the past 12-months period, giving an average of 205 transactions for each of the PowerSellers every month. There were 60,675 unique buyers altogether and we further found that there were 64,267 unique buyers to the 31 PowerSellers combinations. Thus, we know that $15.67 \%$ of the overall transactions were repeat transactions.

From the same set of data, we also found that $87.44 \%$ of all seller-buyer pairs only had a single transaction conducted during that period, while the balance of $12.56 \%$ from the unique buyer-seller pairs had completed at least one repeat transaction between themselves. These two findings indicate how significantly the repetitive transactions had really contributed to the overall feedback score and it would be inappropriate for us not to consider such significant representation.

Besides this, out of the total of 237 negative feedbacks given to all 31 PowerSellers, there were 216 unique buyers who actually made the complaints. Out of these, only 15 of them had actually given both positive and negative feedback towards the same seller. This in a way shows that most of the $93 \%$ of cases came from strangers to that particular seller, as there was no previous history between them at all.

Furthermore, if we observe the last time these positive and negative feedbacks were left, $68.8 \%$ of the positive feedbacks were given prior to the negative, meaning that most of the time, once a negative feedback was left, it will deter the same buyer buying from the same seller again in the future. This result supports the approach that we have taken to let pending conciliation offset the repeated collaboration times and to allow the cancellation of a negative feedback in case reconciliation has been reached, paving the way for their future collaboration.

By using the eBay feedback dataset as a case study, we have been able to highlight some key features and the importance of the proposed trust model. The collective trust measurement (i.e., c index) can be very practical when there is not enough personal experience to begin with or when a robust collective experience from the whole community is needed for collaborator selection. At the same time, the optimal selection method has not compromised on the quality of the potential partner as it still seeks the best possible partner as in any other trust model. The only difference is that the selection is based on one's own standing rather than on the best in the community.

When compared to the more diverse results that we obtained from the eBay feedback score, the c index brings a much more focused and coherent platform that we can use to facilitate collaborators in evaluating collaborative performance, reflecting both the depth and breadth of the trust performance. From this comparison, it is clear that the $\mathrm{c}$ index offers added functionality to classify the PowerSellers into distinct categories, something that is not possible to achieve with the eBay feedback score. Nevertheless, as the $\mathrm{c}$ index and the eBay feedback score are emphasising different attributes, comparing the results will be insignificant for this study.

Employing the c index as a collaborator selection criterion is particularly practical when the two parties do not know each other (i.e., do not have a collaboration history) and need to see if they have a compatible trust perception towards each other. 


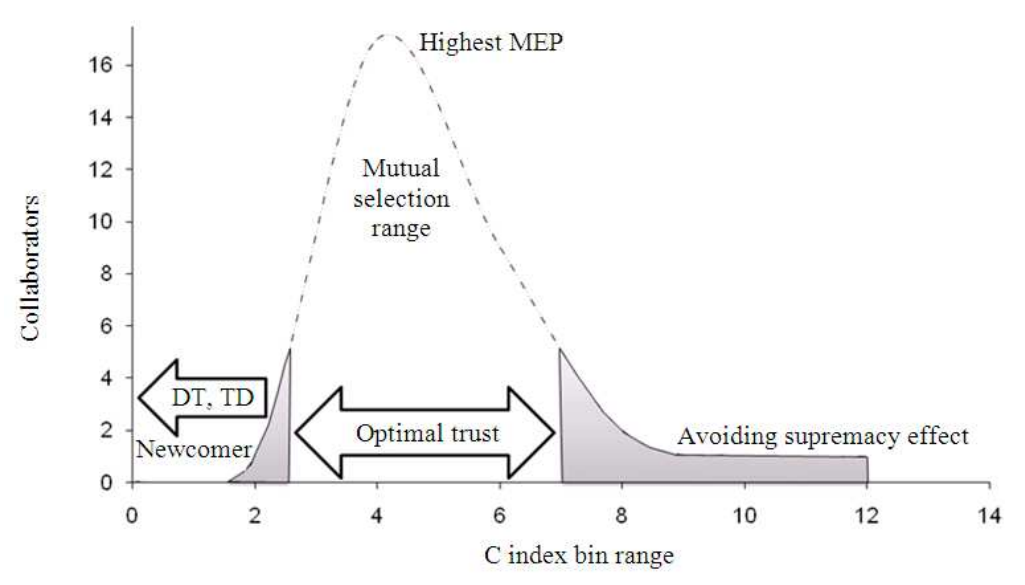

Fig. 2: Frequency of $\mathrm{C}$ index showing the desirable optimal range

From the above we can see that the $\mathrm{c}$ index can produce the needed result that enables us to facilitate potential collaborators in identifying an optimal trust level in the selection process.

Lastly, we refer to the bell-shaped histogram in Fig. 2 that shows the frequency of the $c$ index among the 31 PowerSellers that exhibit common characteristics of natural variation in a normal distribution. More specifically, we have a small number in the lower "tail" that represents the newcomers in the community and the large portion in the middle, as well as another small upper "tail" at the other end that represents the type of supremacy effect that we are trying to avoid.

The simple quality of this normal distribution applies in situations where extreme values on both "tails" are less likely to occur than the "middle range" average values, hence the applicability of the proposed measurement are well justified. For that reason, the optimal trust model can be utilised to facilitate newcomers in identifying collaborators, for instance, to use DT or TD as measurements and continue building their reputation. At the same time this model can also be used to prevent the supremacy effect accordingly as the selection is based on one's own standing, rather than on finding the one with the highest value of the reputation measurement.

\section{CONCLUSION}

We have discussed the concept of optimal trust in collaborative ventures and the importance of such a concept in partner selection processes. By associating this conceptual thought with a collaborative trust framework, we have developed a computational optimal trust model that can be used to facilitate the collaborator selection procedure.

In doing so, we present a trust and reputation model that incorporates two novelties with respect to current models. First, we introduce a new trust measure named the c-index, inspired by the h-index and the Page Rank that uses the number of repeated collaborations to evaluate partners instead of the more common positive feedback score. Secondly, based on this c-index, we propose a new method in selecting collaboration partners that provides emphasis on the mutual aspect of trust.

To the best of our knowledge, there are no other reputation-based computational trust models that have placed mutual interdependencies between collaborators as the underpinning concept in a trust model. Furthermore, based on the collaborative trust framework, we have developed a model, which:

- Provides computational definitions for Direct Trust (DT), Perceived Reputation (PR), Trust Depth (TD), Trust Breadth (TB) and the c index. DT and TD captures the individual trust experience, while PR and c index operate as collective trust (i.e., reputation) measurements and

- Proposes an optimal collaborator selection method that takes one's own individual or collective trust measurement value as a relative threshold to identify potential collaborators that share a mutual trust (i.e. a significant and compatible level of trust towards each other)

In order to analyze the applicability of the trust measurement, we have collected a dataset of over 70,000 feedback scores on 31 of eBay's PowerSellers. From the study that we have conducted, the findings indicate that the $\mathrm{c}$ index as a collective trust measurement provides the essential features to meaningfully classify a large community into identifiable trust categories and thus provide an important basis to facilitate the mutual selection process. This contrasts with the eBay feedback scores 


\section{J. Computer Sci., 8 (9): 1531-1540, 2012}

that spread into a large range and therefore cannot provide a meaningful comparison between the PowerSellers. Nevertheless, at the same time it still provides a much higher standard and more robust collective trust measurement. The eBay dataset experiments and examples have demonstrated the significance and applicability of our trust model in supporting reputation-based collaborative trust measurement in electronic communities.

\section{REFERENCES}

Sabater, J. and C. Sierra, 2005. Review on computational trust and reputation models. Art. Artif. Intell. Rev., 24: 33-60. DOI: 10.1007/s 10462-004-0041-5

Marsh, S.P., 1994. Formalizing trust as a computational concept. Ph.D Thesis, University of Stirling.

McKnight, D.H. and N.L. Chervany, 2001. The meanings of trust. Trust in Cyber-Societies-LNAI, 2246: 27-54.

Bonatti, P., C. Duma, D. Olmedilla and N. Shahmehri, 2005. An integration of reputation-based and policy-based trust management. Proceedings of the Sematic Web and Policy Workshop, (SWPW' 05), Galway, Ireland.

Ramchurn, S.D., D. Huynh and N.R. Jennings, 2004a. Trust in multi-agent systems. Knowl. Eng. Rev., 19: 1-25. DOI: 10.1017/S0269888904000116

Ramchurn, S.D. C. Sierra, L. Godo and N.R. Jennings, 2004b. Devising a trust model for multi-agent interactions using confidence and reputation. Int. J. Appl. Art. Int., 18: 833-852. DOI: 10.1080/0883951049050904509045

Hosmer, L., 1995. Trust: The connecting link between organizational theory and philosophical ethics. Academy Manag. Rev., 20: 379-403.

Wicks, A., S. Berman and T. Jones, 1999. The structure of optimal trust: Moral and strategic implications. Academy Manag. Rev., 24: 99-116.

OD, 2009. Optimal. Oxford University Press.

Dictionary.com. 2009. Optimal. Dictionary.com, LLC.

Kreps, D.M. and R. Wilson, 1982. Reputation and imperfect information. J. Economic Theory, 27: 253-279.

Mokhtar, M.R., U. Wajid and W. Wang, 2007. Collaborative trust in multi-agent system. Proceedings of the 16th IEEE International Workshops on Enabling Technologies: Infrastructures for Collaborative Enterprises, Jun. 18-20, ACM Press, USA., pp: 30-34. DOI: 10.1109/WETICE.2007.130
Winer, M.B. and K.L. Ray, 1994. Collaboration Handbook: Creating, Sustaining and Enjoying the Journey. 1st Edn., Amherst H. Wilder Foundation, Saint Paul, ISBN-10: 0940069032, pp: 178.

Brainov, S. and T. Sandholm, 1999. Contracting with uncertain level of trust. Comput. Intell., 18: 501514. DOI: 10.1111/1467-8640.00200

Yu, B. and M.P. Singh, 2002. Distributed reputation management for electronic commerce. Comput. Intell., 18: 535-549. DOI: 10.1111/14678640.00202

Fan, M., Y. Tan and A.B. Whinston, 2005. Evaluation and design of online cooperative feedback mechanisms for reputation management. IEEE Trans. Knowl. Data Eng. 17: 244-254. DOI: 10.1109/TKDE.2005.26

Vasalou, A., A. Hopfensitz and J.V. Pitt, 2008. In praise of forgiveness: Ways for repairing trust breakdowns in one-off online interactions. Int. J. Human-Comput. Stud., 66: 466-480. DOI: 10.1016/j.ijhcs.2008.02.001

Teacy, W.T.L., J. Patel, N.R. Jennings and M. Luck, 2006. TRAVOS: Trust and reputation in the context of inaccurate information sources. Autonomous Agents Multi-Agent Syst., 12: 183-198. DOI: 10.1007/s10458-006-5952-x

Resnick, P. and R. Zeckhauser, 2002. Trust among strangers in internet transactions: Empirical analysis of eBay' s reputation system. Econ. Internet E-Commerce, 11: 127-157. DOI: 10.1016/S0278-0984(02)11030-3

Sabater, J. and C. Sierra, 2001. REGRET: Reputation in gregarious societies. Proceedings of the 5th International Conference on Autonomous Agents, May 28-Jun. 1, ACM Press, USA, pp: 194-195. DOI: $10.1145 / 375735.376110$

Hirsch, J.E., 2005. An index to quantify an individual's scientific research output. PNAS, 102: 1656916572. DOI: $10.1073 /$ pnas.0507655102

Dellarocas, C., 2000. Immunizing online reputation reporting systems against unfair ratings and discriminatory behavior. Proceedings of the 2nd ACM Conference on Electronic Commerce, (EC' 00), ACM Press, USA., pp: 150-157. DOI: $10.1145 / 352871.352889$

Mozenda, 2008. Mozenda web data on demand. Mozenda Inc. 